Jean Dubé Louis-Philippe Boulet

\section{ADRESSE}

J. Dubé: maître ès sciences. L.P. Boulet: docteur en médecine, $F R C P(c)$. Unité de recherche, centre de pneumologie de l'hôpital Laval, universite Laval, 2725 Chemin Sainte-Foy, Sainte-Foy, Québec, G1V 4G5, Canada.

\title{
Rôles de l'inflammation et des modifications des structures bronchiques dans I'asthme allergique
}

L'exposition à des allergènes communs peut provoquer chez des sujets sensibilisés l'apparition d'un asthme bronchique symptomatique. La modification de la réactivité bronchique semble secondaire à une réaction inflammatoire impliquant des cellules et des substances chimiotactiques et à des altérations de la structure bronchique, avec atteinte de l'épithélium, dépôt de collagène sous la membrane basale et hyperplasie des fibres musculaires lisses. Les mastocytes amorceraient ce processus, alors que les éosinophiles et les lymphocytes seraient impliqués dans l'établissement et la persistance de l'inflammation asthmatique. Certaines modifications de la matrice extracellulaire de la paroi bronchique, induites par le processus inflammatoire, sont peut-être responsables de l'irréversibilité de l'hyperréactivité bronchique, aggravant les conséquences des poussées d'inflammation aiguë provoquées par le contact réitéré avec les allergènes et les surinfections.

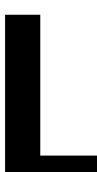

'asthme est une maladie qui se manifeste par des épisodes répétés d'obstruction bronchique et une hyperréactivité des bronches aux stimuli endogènes ou exogènes [1]. Ces phénomènes sont généralement considérés comme secondaires à une réaction inflammatoire des voies aériennes, caractérisée par une infiltration des bronches par des cellules activées, un odème et une hypersécrétion bronchique $[2,3]$. Bien que la relation exacte entre cette inflammation et l'expression clinique ou physiopathologique de la maladie reste à préciser, certaines modifica- tions de la structure bronchique, vraisemblablement déclenchées par cette réaction inflammatoire, semblent jouer un rôle dans l'apparition et la persistance de l'asthme [4]. Parmi ces modifications, il faut mentionner le dépôt de collagène sous l'épithélium, les altérations de la matrice extracellulaire bronchique, l'atteinte de l'épithélium et l'hyperplasie des fibres musculaires lisses des bronches.

La réaction allergique est considérée comme une manifestation exagérée ou inappropriée d'un mécanisme de défense de l'organisme contre les agressions extérieures. L'améliora- 
tion et l'utilisation plus répandue des techniques endoscopiques ont permis, au cours des dernières années, d'étudier plus à fond l'inflammation de la muqueuse respiratoire dans diverses situations, en particulier lors du phénomène allergique, découlant de l'exposition naturelle ou expérimentale aux allergènes communs [5].

\section{Les réactions allergiques asthmatiques immédiates et tardives}

L'inhalation d'un allergène par un sujet sensibilisé peut provoquer divers types de réaction bronchique, les deux principaux étant les réactions "immédiate » et "tardive » (figure 1). La réaction "immédiate» se manifeste par une diminution des débits expiratoires et l'apparition de symptômes d'asthme, comme la dyspnée, la sensation d'oppression thoracique, les sillements et la toux, et ce durant la première heure après l'exposition à l'allergène. La réaction asthmatique immédiate est principalement due à une contraction des muscles lisses bronchiques et à un œdème de la muqueuse en réponse à des médiateurs d'origine principalement mastocytaire [6]. Cette réaction s'estompe spontanément, habituellement en moins d'une heure.

Par la suite, certains sujets peuvent présenter une deuxième réaction asthmatique. Cette réaction, appelée tardive, apparaît trois à huit heures après l'inhalation de l'allergène [7]. Elle s'accompagne d'une inflammation bronchique caractérisée par une augmentation du nombre de lymphocytes et d'éosinophiles mise en évidence par l'analyse du liquide de lavage bronchoalvéolaire (LBA) [8-10]. Chez les asthmatiques qui présentent une réaction tardive, il existe des corrélations variables mais significatives entre l'intensité de cette réaction et l'amplitude de la réaction immédiate, la quantité d'allergène inhalée, la réactivité immunologique du sujet à cet allergène et la réactivité non allergique de ses voies aériennes [11]. Il semble d'ailleurs que tout asthmatique peut avoir une réaction tardive s'il inhale des quantités suffisantes d'allergène, en utilisant un bronchodilatateur à courte durée d'action avant et pendant la provocation antigénique pour inhiber la réaction immédiate [12].

\section{Les cellules et les médiateurs impliqués dans I'inflammation bronchique (figure 1)}

\section{Les mastocytes}

Considérés autrefois comme les principales cellules mises en cause dans l'asthme, les mastocytes jouent un rôle encore discuté dans la réaction asthmatique allergénique. Ils semblent pourtant nombreux dans le liquide de LBA et l'épithélium bronchique des asthmatiques; ces derniers présentent, par ailleurs, des taux plus élevés d'histamine, de tryptase et de prostaglandine $\mathrm{D}_{2}\left(\mathrm{PgD}_{2}\right)$ dans le liquide de LBA que les sujets normaux. Outre son rôle probable dans la genèse de la réaction immédiate, le mastocyte pourrait également contribuer à l'inflammation allergique. L'activation de mastocytes par stimulation antigénique des récepteurs FceR I entraîne la production de leucotriènes (LT), du facteur de stimulation des colonies de granulocytes et de macrophages (GMCSF), et des interleukines (IL) 3,4 , 5 et 6 [13]. Ces cytokines influencent la mobilisation, l'attraction et l'activation des neutrophiles, des monocytes et des éosinophiles. Par la synthèse in vivo de ces cytokines, les mastocytes pourraient participer indirectement à la réaction asthmatique tardive chez l'homme [14].

\section{Les macrophages}

Le macrophage, qui a des propriétés de cellule présentatrice d'antigène, a le potentiel de libérer de nombreux médiateurs capables d'induire un ou plusieurs des éléments inflammatoires retrouvés dans la réaction asthmatique allergique. Des récepteurs d'IgE de faible affinité (FcER II) retrouvés à sa surface peuvent causer son activation à la suite de son exposition à un allergène [15]. Une fois activé, le macrophage libère divers médiateurs lipidiques comme le facteur d'activation des plaquettes (PAF) [16], le leucotriène $\mathrm{B} 4$, la PGF2 $\alpha$ et la thromboxane B2. Ces médiateurs agiraient en synergie avec ceux du mastocyte pour amplifier la réaction asthmatique immédiate.

Le macrophage semble également 


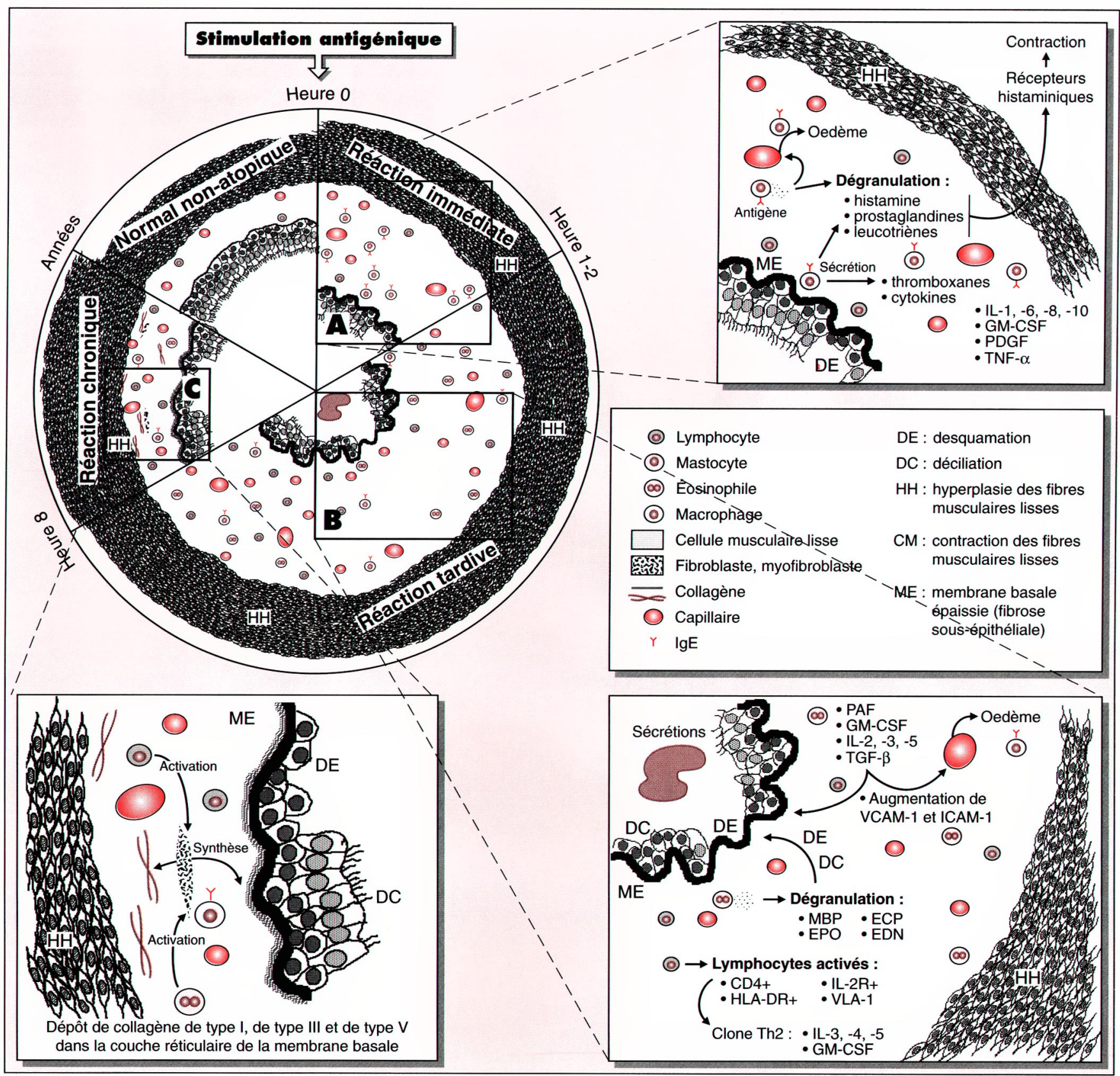

Figure 1. Chronologie de la réaction asthmatique. On distingue trois périodes dans la réaction asthmatique à un allergène : $\boldsymbol{A}$ la réaction immédiate, $\boldsymbol{B}$ la réaction tardive, une à huit heures plus tard, $\boldsymbol{C}$ la réaction chronique au bout de plusieurs années. Principaux changements cellulaires, inflammatoires et structuraux bronchiques observés après une stimulation antigénique. A. Réaction immédiate : I'allergène (antigène) réagit avec les récepteurs FceRl des mastocytes et les récepteurs FceRll des macrophages, ce qui entraîne le relargage d'histamine qui va stimuler les muscles bronchiques et produire une bronchoconstriction. Les cellules du système immunitaire relarguent les médiateurs de l'inflammation allergique : prostaglandines, leucotriènes, thromboxanes, cytokines, responsables de I'œdème. B. Réaction tardive: elle apparaît quelques heures après l'inhalation d'allergènes. L'inflammation bronchique est caractérisée par une augmentation du nombre des lymphocytes et des éosinophiles. Certaines cytokines agissent sur l'endothélium et l'épithélium bronchiques et augmentent la synthèse de molécules d'adhérence, telles VCAM-1 et ICAM-1 qui mobilisent les éosinophiles vers le poumon. Les éosinophiles relarguent leurs granulations riches en protéines cytotoxiques (MBP major basic protein, EPO eosinophil peroxidase, EDV eosinophil-derived neurotoxin, ECP eosinophil cationic protein). Les cytokines sécrétées par les lymphocytes pourraient expliquer l'apparition de l'inflammation chronique. Le rôle des neutrophiles est plus discutable. C. Réaction chronique : probablement secondaires au processus inflammatoire, on observe des modifications morphologiques de la paroi bronchique : dépôt de collagène et épaississement de la membrane basale, hyperplasie et hypertrophie du muscle lisse bronchique. 
18. Johnston SL, Holgate ST. The inflammatory response in asthma. Br J Hosp Med $1991 ; 46: 84-90$.

19. Wegner CD, Gundel RH, Reilly P, Haynes N, Letts LG, Rothlein R. Intercellular adhesion molecule-1 (ICAM-1) in the pathogenesis of asthma. Science $1990 ; 247$ : 456-9.

20. Gonzalez MC, Diaz P, Galleguillos FR, Ancic P, Cromwell O, Kay AB. Allergen-induced recruitment of bronchoalveolar Helper (OKT4) and Suppressor (OKT8) T-cells in asthma. Am Rev Respir Dis 1987; 136: 6004.

21. Gerblich AA, Cambell AE, Schuyler MR. Changes in T-lymphocytes subpopulations after antigenic bronchial provocation in asthmatics. N Engl J Med 1984 ; 310 : 134952.

22. Wilson JW, Djukanovic R, Howarth PH, Holgate ST. Inhaled beclomethasone diproprionate downregulates airway lymphocyte activation in atopic asthma. Am J Respir Crit participer à la mise en branle de la réaction asthmatique tardive par la sécrétion de médiateurs et de cytokines pro-inflammatoires tels l'IL-1, l'IL-6, le GM-CSF, le facteur de croissance dérivé des plaquettes (PDGF), le facteur nécrosant des tumeurs $\alpha$ (TNF- $\alpha$ ), et des facteurs de libération de l'histamine [3, 14, 17]. Le GM-CSF peut agir sur les éosinophiles en stimulant leur fonction inflammatoire et en provoquant une activation des progéniteurs des éosinophiles [18].

Le passage des cellules inflammatoires du sang dans le poumon s'effectue en réponse aux différentes cytokines et médiateurs chimiotactiques $[14,19]$. Cette migration cellulaire coincide habituellement avec le début de la réaction asthmatique tardive, qui s'accompagne d'une augmentation du nombre des éosinophiles hypodenses dans le sang [9]. Différentes cytokines (principalement l'IL-2, IL-3, IL-5, GM-CSF) relâchées lors de la réaction asthmatique agissent sur l'épithélium et l'endothélium bronchiques en augmentant l'expression des molécules d'adhérence (VCAM-1, ICAM-1), qui sont extrêmement importantes pour la mobilisation des éosinophiles vers le poumon $[10,14,18]$. Plusieurs données laissent croire que l'éosinophile, tout comme le lymphocyte, est essentiel à l'expression des réactions allergiques et joue un rôle majeur dans la mise en place de l'hyperréactivité bronchique d'origine allergique.

\section{Les lymphocytes}

réduit l'expression de ces marqueurs cellulaires, en relation avec une amélioration clinique [22].

Les lymphocytes activés ont probablement un rôle à jouer dans l'élaboration de la réaction asthmatique chronique, et ce grâce à différentes modalités de sécrétion des lymphokines. Il existe deux principaux clones de lymphocytes T. Les lymphocytes auxiliaires ( $T$ helper, Th)Th1 sont responsables de la sécrétion de l'IL-2 et de l'interféron $\gamma($ IFN- $\gamma)$ et les Th2, de la sécrétion de l'IL-3, de l'IL-4, de l'IL-5 et du GM-CSF [13, 2123]. L'IL-4 et l'IFN- $\gamma$ règlent la synthèse des IgE par les lymphocytes $\mathrm{B}$, le premier ayant des effets de stimulation et le second d'inhibition [23-25]. L'IL-3, l'IL-5 et le GM-CSF agissent pour leur part sur le recrutement et l'activation des éosinophiles, et l'IL-3 et l'IL-4 activent les mastocytes et les basophiles.

Malgré la sécrétion de cytokines par les mastocytes et les fibroblastes dans la réaction asthmatique tardive, l'activation et l'augmentation du nombre de lymphocytes $\mathrm{T}$ de type Th2 privilégient cette cellule comme principal modulateur de l'inflammation asthmatique [23, 26]. Le développement d'un clone de type Th2 et l'effet de leurs cytokines sur les autres cellules inflammatoires et les cellules constituantes de la muqueuse bronchique pourraient expliquer l'apparition de l'inflammation chronique et de l'hyperréactivité retrouvées dans l'asthme.

\section{Les éosinophiles}

La réaction asthmatique tardive s'accompagne d'une mobilisation des lymphocytes. Chez les sujets asthmatiques allergiques, les proportions des différents types de lymphocytes $\mathrm{T}$ changent dans le LBA et le sang après une bronchoprovocation allergénique [20]. Ce fait suggère que les lymphocytes $\mathrm{T}$ jouent un rôle dans la régulation de la réaction asthmatique tardive. La mobilisation sélective dans le poumon de lymphocytes $\mathrm{T}$ $\mathrm{CD} 4^{+}$, exprimant des marqueurs d'activation tels que HLA-DR, IL-2R (récepteur de l'interleukine 2) et VLA-1 (very late activation antigen 1), accompagnerait le développement de la réaction asthmatique tardive [21]. L'administration de corticostéroïdes prévient la réaction tardive et
Les granulations de l'éosinophile contiennent des protéines riches en arginine, comme la protéine basique majeure (MBP), la peroxydase de l'éosinophile (EPO), la neurotoxine dérivée de l'éosinophile (EDN) et la protéine cationique de l'éosinophile (ECP) $[18,22]$. Ces protéines possèdent des propriétés cytotoxiques pour l'épithélium respiratoire et peuvent être responsables, du moins en partie, des lésions épithéliales observées dans l'asthme symptomatique. La réaction asthmatique allergique tardive s'accompagne d'ailleurs d'une accumulation d'éosinophiles activés positifs pour EG2 (l'EG2 est un anticorps reconnaissant un antigène de l'ECP sécrétée) et de la pré- 
sence des produits des granulations dans le liquide de lavage bronchoalvéolaire [27].

L'éosinophile peut aussi contribuer à la pathogénie de l'asthme par la synthèse de médiateurs lipidiques (leucotriène C4) et de cytokines GM-CSF, IL-2, IL-3, IL-5, IL-6, TGF- $\beta$ (transforming grouth factor $\beta$ ), TNF- $\alpha$, et PAF dont les rôles ont été décrits plus haut [28-34]. Le PAF a un effet chimiotactique et augmente l'adhérence de l'éosinophile en plus de causer une vasodilatation, une augmentation de la perméabilité vasculaire et de la sécrétion bronchique, ainsi qu'une hyperréactivité bronchique quand il est inhalé [27, 34].

23. Robinson DS, Hamid O, Ying S, Tsicopoulos A, Barkans J, Bentley AM, Corrigan C, Durham SR, Kay AB. Predominant Th2-like bronchoalveolar T-lymphocyte population in atopic asthma. $N$ Engl J Med 1992; $326: 298-304$.

24. Banchereau J. Interleukine-4. médecine/sciences $1990 ; 6$ : 946-53.

25. Holgate ST. Mediator and cytokine mechanisms in asthma. Thorax 1993; 48: 103-9.

26. Holgate ST, Djukanovic R, Howarth PH, Montefort S, Roche W. The T cell and the airway's fibrotic response in asthma. Chest $1993 ; 103: 125 \mathrm{~S}-8$

27. Kay AB. Asthma and inflammation. $J A l$ lergy Clin Immunol 1991 ; 87 : 893-910.

28. Kita H, Ohnishi T, Okubo Y, Weiler D, Abrams JS, Gleich GJ. Granulocyte/macrophage colony-stimulating factor and interleukin-3 release from human peripheral blood eosinophils and neutrophils. I Exp Med $1991 ; 174$ : 745-8.

29. Broide DH, Paine MM, Firestein GS. Eosinophils express interleukin 5 and Granulocyte Macrophage-Colony-stimulating factor mRNA at sites of allergic inflammation in asthmatics. J Clin Invest 1992; 90 : 141424.

30. Hamid Q, Barkans J, Meng Q, Ying S, Abrams JS, Kay AB, Moqbel R. Human eosinophils synthesize and secrete interleukin-6, in vitro. Blood 1992 ; 80 : 1496-501.

31. Costa II, Matossian K, Resnick MB, Beil WJ, Wong DT, Gordon JR, Dvorak AM, Weller PF, Galli SJ. Human eosinophils can express the cytokine tumor necrosis factor-alpha and macrophage inflammatory protein-1 alpha. J Clin Invest 1993; 91 : 2673-84.

32. Wong DTW, Weller PF, Galli SI, Elovic A, Rand TH, Gallagher GT, Chaing T, Chou MY, Matossian K, McBride J, Todd R. Human eosinophils express transforming growth factor $\alpha . J$ Exp Med $1990 ; 172$ : 673-81.

33. Ohno I, Lea RG, Flanders KC, Clark DA, Banwatt D, Dolovich J, Denburg J, Harley CB, Gauldie J, and Jordana M. Eosinophils in chronically inflamed human upper airway tissues express transforming growth factor $\beta 1$ gene (TGF $\beta 1$ ). J Clin Invest 1992; 89 : $1662-8$.

$m / s n^{\circ} 3$, vol. 12 , mars 96
Il faut savoir cependant que l'inflammation bronchique en soi ne cause pas toujours un asthme ou une hyperréactivité bronchique. A cet égard, nous avons récemment démontré la présence d'inflammation bronchique marquée chez des sujets non asthmatiques ayant une toux chronique ou une rhinite allergique [37, 38]. L'inflammation observée dans ces cas a plusieurs points de similitude avec celle observée dans l'asthme allergique; cependant, les sujets asthmatiques présentent une fibrose sous-épithéliale, alors que les sujets qui ont une toux chronique n'en présentent aucune et ceux qui souffrent de rhinite ont une fibrose moins marquée et focale. On a démontré que la fibrose sous-épithéliale est principalement due au dépôt de collagène de type I, de type III et de type V ainsi que de fibronectine provenant probablement de myofibroblastes présents dans la paroi bronchique [39, 40]. Nous avons trouvé pour notre part une excellente corrélation entre l'intensité du dépôt de collagène (voir figure 3), tel que mis en évidence par la mesure de l'épaisseur apparente de la membrane basale en microscopie optique, et le niveau de la réactivité bronchique [37].

La réponse des bronches aux agonistes est peut-être augmentée par des facteurs modifiant l'épaisseur ou les propriétés mécaniques de la paroi bronchique, comme la collagénisation, l'œdème de l'adventice entrấnant une réduction de la postcharge du muscle lisse bronchique, la présence de collagène à travers les fibres musculaires lisses et certains changements vasculaires [41]. Ces modifications bronchiques peuvent provoquer des modifications du tonus bronchomoteur, de la réponse bronchique aux stimuli et de la réaction maximale des voies aériennes.

Nous disposons cependant de plus d'hypothèses que de réponses, et plusieurs points restent à préciser pour établir une relation de cause à effet entre la réaction inflammatoire bronchique et les remaniements de l'architecture présents en cas d'hyperexcitabilité bronchique. A ce sujet, nous avons récemment mis au point une technique d'isolement des fibroblastes d'asthmatique à partir de biopsies bronchiques [42]. L'obten- 


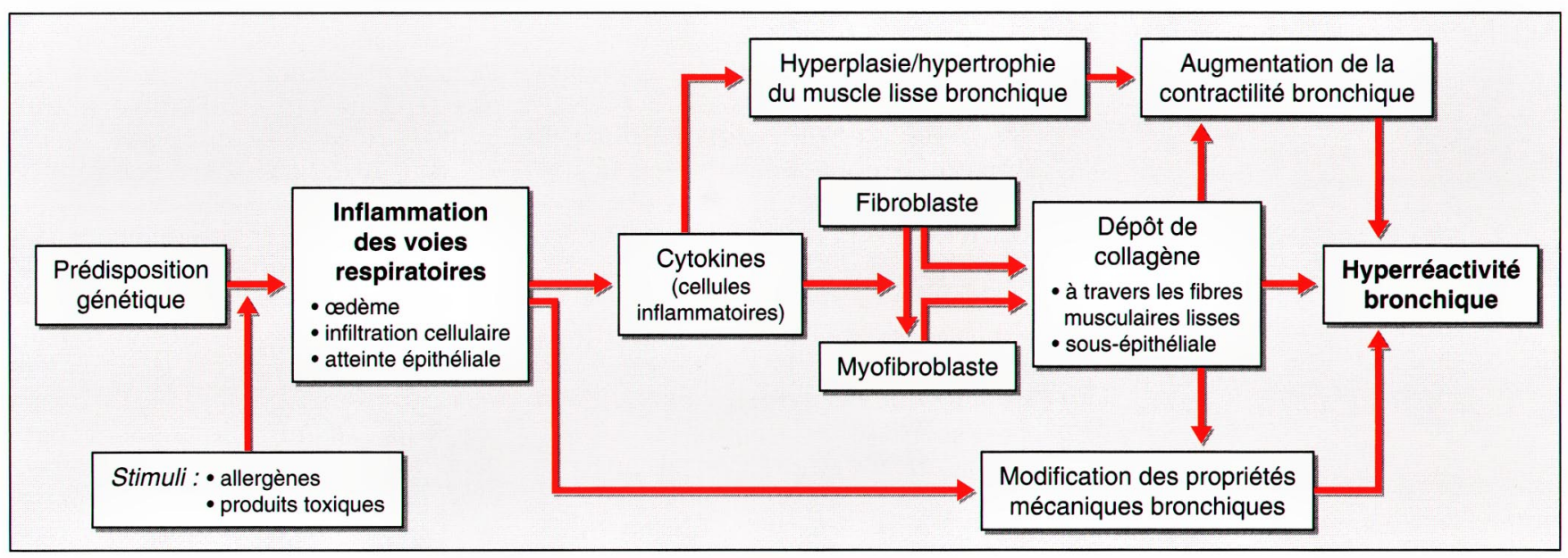

Figure 2. Physiopathogénie de l'asthme allergique: de l'inflammation à I'hyperréactivité bronchique.
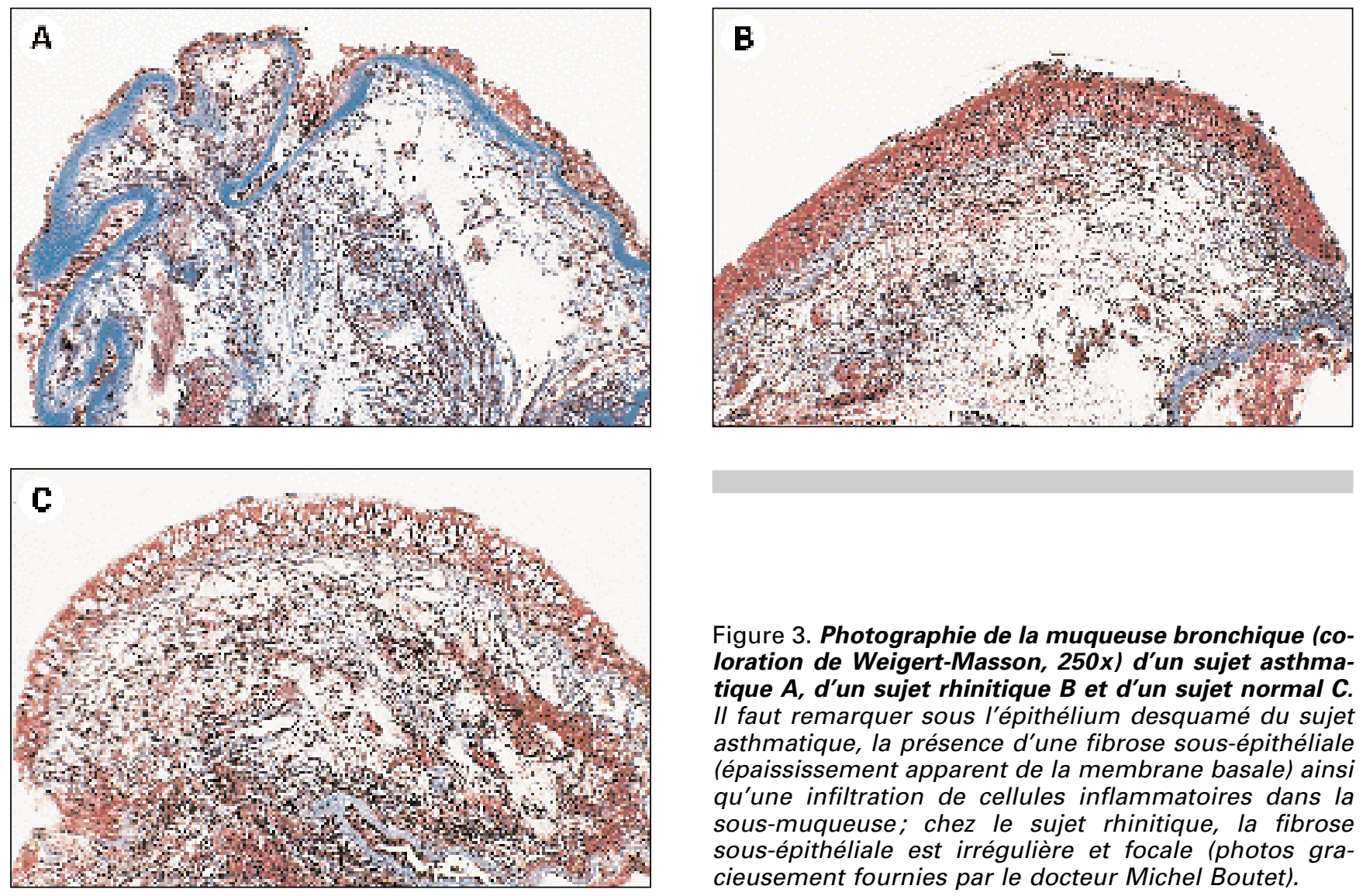

Figure 3. Photographie de la muqueuse bronchique (coloration de Weigert-Masson, 250x) d'un sujet asthmatique $A$, d'un sujet rhinitique $B$ et d'un sujet normal $C$. Il faut remarquer sous l'épithélium desquamé du sujet asthmatique, la présence d'une fibrose sous-épithéliale (épaississement apparent de la membrane basale) ainsi qu'une infiltration de cellules inflammatoires dans la sous-muqueuse; chez le sujet rhinitique, la fibrose sous-épithéliale est irrégulière et focale (photos gracieusement fournies par le docteur Michel Boutet).

tion de ce type de matériel permettra l'étude des interactions entre le processus inflammatoire et le fibroblaste, cellule considérée comme une des principales responsables des modifications de la structure bronchique chez l'asthmatique. Il est possible que les lésions chroniques, tel le dépôt de

soient responsables de la chronicité de l'hyperexcitabilité bronchique, certains facteurs tel l'œedème pouvant modifier transitoirement ce niveau de réactivité. Il est aussi possible qu'en phase précoce de la maladie seuls les facteurs inflammatoires influencent la réactivité bronchique, et qu'avec l'installation des lésions chroniques il y ait avec le temps persistance de l'hyperréactivité bronchique. Le peu d'effet des corticostéroïdes à corriger les lésions chroniques des bronches comme le dépôt de collagène plaide en faveur de cette hypothèse $[43,44]$. Il est donc important de mieux préciser la nature et les conséquences des anomalies inflammatoires et structu- 
rales retrouvées dans l'asthme afin de définir leurs rôles dans l'installation de l'asthme. Il n'existe pour l'instant aucun traitement qui corrige les anomalies de la structure bronchique retrouvées dans cette maladie. La réduction des contacts allergiques et le traitement précoce de l'inflammation bronchique, particulièrement avec les corticostéroïdes, constituent donc pour l'instant les moyens les plus efficaces de prévention et d'atténuation des lésions bronchiques retrouvées dans l'asthme

\section{RÉFÉRENCES}

34. Cuss FM, Dixon CM, Barnes PJ. Effetcs of inhaled platelet-activating factor on pulmonary function and bronchial responsiveness in man. Lancet 1986 ; 2 : 189-92.

35. O'Byrne P. Neutrophil dynamics in airway disease. In : Busse WW, Holgate ST, eds. Asthma and rhinitis. Boston: Blackwell Scientific Publications, 1995 : 389-96.

36. Moreno RH, Hogg JC, Paré PD. Mechanics of airway narrowing. Am Rev Respir Dis 1986 ; 133 : 1171-80.

37. Boulet LP, Turcotte H, Boutet M, Milot J, Côté, J, Malo JL, Cartier A, Dugas M, Laviolette M. Correlations between airway inflammation and responsiveness to methacholine in subjects with asthma, rhinitis, reactive airway dysfunction syndrome, chronic cough and normals. Eur Resp J 1993 : 6 (suppl 17) : 265S

38. Boulet LP, Milot J, Boutet M, St-Georges F, Laviolette M. Airway inflammation in non-asthmatic subjects with chronic cough. Am J Respir Crit Care Med 1994 ; 149 : 482-9.

39. Roche WR, Beasley R, Williams JH, Holgate ST. Subepithelial fibrosis in the bronchi of asthmatics. Lancet $1989 ; 1$ : 520-4.

40. Roche WR. Fibroblasts and asthma. Clin Exp Allergy 1991 ; 21 : 545-8.

41. James A. Limited airway narrowing : why doesn't everyone have asthma? Eur Respir J 1994 ; 7 : 1210-2.

42. Goulet F, Auger FA, Germain L, Dubé J, Laviolette M, Boutet M, Boulet LP. Isolation of epithelial and fibroblastic cells from bronchial biopsies (BB) of asthmatic subjects. Am J Resp Crit Care Med 1994; 149: A631.

43. Jeffery PK, Godfrey RW, Ädelroth E, Nelson F, Rogers A, Johansson SA. Effects of treatement on airway inflammation and thickening of basement membrane reticular collagen in asthma. Am Rev Respir Dis 1992 ; $145: 890-9$

44. Trigg CJ, Manolitsas ND, Wang J, et al. Placebo-controlled immunopathologic study of four months of inhaled corticosteroids in asthma. Am J Respir Crit Care Med 1994 ; $150: 17-22$

$m / s n^{\circ} 3$, vol. 12 , mars 96

\section{Summary}

The role of bronchial inflammation and structural modifications in allergic asthma

Exposure to common allergens can induce symptomatic asthma in sensitized individuals. The processes by which allergens modify airway function are still unknown but could be secondary to bronchial inflammation following the production and release of chemotactic and phlogistic mediators by inflammatory cells. The mast cell seems to initiate this process, but eosinophils and lymphocytes are considered responsible for the establishment and persistence of the inflammatory process which leads to alterations of the bronchial structure such as epithelial damage, collagen deposition beneath the basement membrane and smooth muscle hyperplasia. Some modifications of the airway wall content in collagen or other extracellular matrix components can possibly explain the irreversible component of airway hyperresponsiveness sometimes observed, occasionally aggravated by inflammatory outbreaks. Increasing our knowledge of the mechanics involved in post-inflammatory structural modifications could provide some avenues in the search for new treatments of asthma.

\section{TIRÉS À PART}

L.P. Boulet. 